\title{
RELAÇÃO ENTRE O DESENVOLVIMENTO DE CATARATA NOS PACIENTES EM \\ USO DE TAMOXIFENO \\ Categoria Clínico
}

INSTITUIÇÃO: Centro Universitário São Camilo.

AUTORES:Guilherme $\mathrm{FF}^{1}$;

Endereço: Rua Capitão Otávio Machado, 1210, apto 21B

Telefone: (11) 97416-6716

Email: felipefguilherme@gmail.com

Bottura HR ${ }^{1}$;

ORIENTADORES: Raia DA².

Discentes da Faculdade de Medicina do Centro Universitário São Camilo;

Médica dermatologista pela Faculdade de Medicina de Rio Preto - FAMERP. ${ }^{2}$ 
RELAÇÃO ENTRE O DESENVOLVIMENTO DE CATARATA NOS PACIENTES EM USO DE TAMOXIFENO

Categoria Clínico

DESCRITORES: “TAMOXIFEN", "CATARACT”, "DRUG-INDUCED”. 


\section{RESUMO}

INTRODUÇÃO:O Tamoxifeno, uma droga derivada do trifeniletileno, é um antagonista de estrógeno não esteroidal, atuando modulando seletivamente receptores de estrógeno. Foi utilizado pela primeira vez em 1971 e, após comprovada sua eficácia, vem sendo amplamente utilizado como terapia hormonal adjuvante no tratamento do câncer de mama metastático com receptores de estrógeno positivos.

Em 1978, foram constatadas as primeiras alterações oculares causadas pelo fármaco. Desde então, muito se estuda sobre os efeitos da droga e sua relação com as doenças oftalmológicas. Atualmente, já está bem documentada sua correlação com a retinopatia e a ceratopatia, contudo sua ligação com o desenvolvimento de catarata segue sendo controversa.

OBJETIVO:O presente artigo tem como proposta analisar a relação entre a exposição ao Tamoxifeno e o desenvolvimento da catarata que tenham sido relatados na literatura até o atual momento.

MÉTODO:Foi realizada uma pesquisa na base de dados Medline/PubMed através da estratégia de busca com os descritores "Tamoxifen" e "Cataract". Dos 42 resultados obtidos, reduziu-se para nove, ao restringir a procura por artigos contendo informações relevantes sobre o elo entre o uso do tamoxifeno e o desenvolvimento de catarata, sem especificação de grupo, região ou ano de publicação.

RESULTADO:As evidências acerca da relação entre o tamoxifeno e o desenvolvimento de catarata são controversas. Ainda que não unânime, grande parte dos estudos analisados revela uma efetiva opacificação do cristalino predisposta pela terapia antiestrogênica em questão. Alguns autores já tentaram explicar as bases fisiopatológicas do processo, mas não obtiveram resultados categóricos.

CONCLUSÃO:Embora algumas reações oftalmológicas adversas já estejam bem documentadas, a predisposição específica à catarata ainda é um tema divergente. Assim, mais estudos são necessários para a documentação de um resultado seguramente definitivo. 


\section{RELAÇÃO ENTRE O DESENVOLVIMENTO DE CATARATA NOS PACIENTES EM USO DE TAMOXIFENO}

\section{INTRODUÇÃO}

O câncer de mama é a neoplasia maligna mais comum na mulher, sendo a segunda neoplasia maior causadora de morte nessa população, atrás apenas do câncer de pulmão ${ }^{(1)}$. Em geral, a sobrevida em cinco anos, dos cânceres de mama é alta, correspondendo a 98\% nos estágios iniciais, $84 \%$ nos casos localmente avançados e $27 \%$ nos tumores metastáticos ${ }^{(2)}$.

Sabendo-se que o câncer de mama é uma afecção heterogênea e multifatorial, deve-se esperar que diversos fatores sejam analisados ao realizar o diagnóstico, entre eles: o estadiamento, caracterizar o tumor, calcular o prognóstico do paciente e selecionar a melhor modalidade terapêutica. Dentre estes fatores, devem ser destacados a expressão de receptores de estrógeno (ER) e progesterona (PR), expressão do gene receptores para proliferação celular HER2, características anatomopatológicas do tumor, grau de expressão de Ki67 e estadiamento TNM ${ }^{(1,2)}$.

Os tumores que expressam graus relevantes de receptores para estrógeno são, em geral, mais bem diferenciados, cursando com neoplasias mais indolentes e com menores riscos de metástases. Uma vez sabido que os hormônios estrogênios estimulam a proliferação do tecido mamário, uma das modalidades terapêuticas existentes para os tumores com expressão positiva de ER é a hormonioterapia $^{(1,3)}$.

Vários tipos de hormonioterapia já foram desenvolvidas e são utilizadas no tratamento do câncer de mama. Dentre elas, destacam-se os moduladores seletivos do receptor de estrogênio (SERM), inibidores de aromatase e a ablação ovariana ${ }^{(1,2)}$.

Dentro do grupo dos SERMs, há uma relativa notoriedade para o Tamoxifeno, uma droga derivada do trifeniletileno. Trata-se de um antagonista não esteroidal de estrógeno que foi utilizado pela primeira vez em 1971 e, após comprovada sua eficácia, vem sendo amplamente utilizado como terapia hormonal adjuvante no tratamento do câncer de mama metastático com receptores de estrógeno positivos ${ }^{(1,3)}$.

Seus efeitos antiestrogênicos vêm da sua capacidade de se ligar competitivamente nos receptores de estrógeno especificamente mamários, impedindo, assim, a angiogênese tumoral, diminuindo síntese de fatores de crescimento teciduais, como IGF-1 e TGF, entre outros fatores benéficos no impedimento do avanço do tumor. Embora aja de maneira obliteradora nos receptores mamários, são notáveis seus efeitos agonistas nos tecidos ósseo e ovariano, mantendo a densidade mineral óssea e melhorando o perfil lipídico dos pacientes que fazem uso da droga ${ }^{(1,3)}$.

É importante destacar outros medicamentos pertencentes ao grupo dos SERMs, que não são utilizados como primeira linha de tratamento do câncer de mama metastático. O Raloxifeno, por exemplo, tem se mostrado mais eficaz na prevenção de osteoporose e fraturas de vértebras em mulheres pósmenopausa com baixa massa óssea ${ }^{(4)}$. Já o Toremifeno, vem sendo empregado cada vez mais no tratamento do câncer de mama com ER positivo, porém em casos específicos, principalmente nos casos em que o Tamoxiefeno não tem resultado muito positivo. 
Contudo, muitos efeitos adversos já foram descritos ao uso do Tamoxifeno. Dentre eles, os mais comuns são náuseas, fogachos, secura vaginal e perda de libido. Entre os efeitos mais raros encontram-se sintomas de ansiedade, ressecamento e descamação da pele e mucosas, dor mamária, febre, desmaios e outra vasta gama de sintomas, incluindo afecções oculares ${ }^{(3)}$.

Em 1978, foram constatadas as primeiras alterações oculares causadas pelo fármaco. Desde então, muito se estuda sobre os efeitos da droga e sua relação com as doenças oftalmológicas. Atualmente, já está bem documentada sua correlação com a retinopatia e a ceratopatia, porém sua ligação com o desenvolvimento de catarata segue sendo controversa.

\section{OBJETIVOS}

\subsection{OBJETIVO GERAL}

- Analisar a relação entre a exposição ao Tamoxifeno e o desenvolvimento da catarata que tenham sido relatadas na literatura.

\subsection{OBJETIVOS ESPECÍFICOS}

- Relatar as bases fisiopatológicas da possível indução da catarata pelo uso de Tamoxifeno.

- Comparar a taxa de indução de catarata do Tamoxifeno com outras drogas de mesma classe.

\section{METODOLOGIA}

Foi realizada uma pesquisa na base de dados Medline/PubMed através da estratégia de busca com os descritores "Tamoxifen" e "Cataract". Dos 42 resultados obtidos, reduziu-se para nove ao restringir a procura por artigos contendo informações relevantes sobre o elo entre o uso do tamoxifeno e o desenvolvimento de catarata, sem especificação de grupo, região ou ano de publicação.

Fig.1. Fluxograma de metodologia.

PESQUISA NA BASE DE DADOS PubMED

"Tamoxifen AND Cataract" 


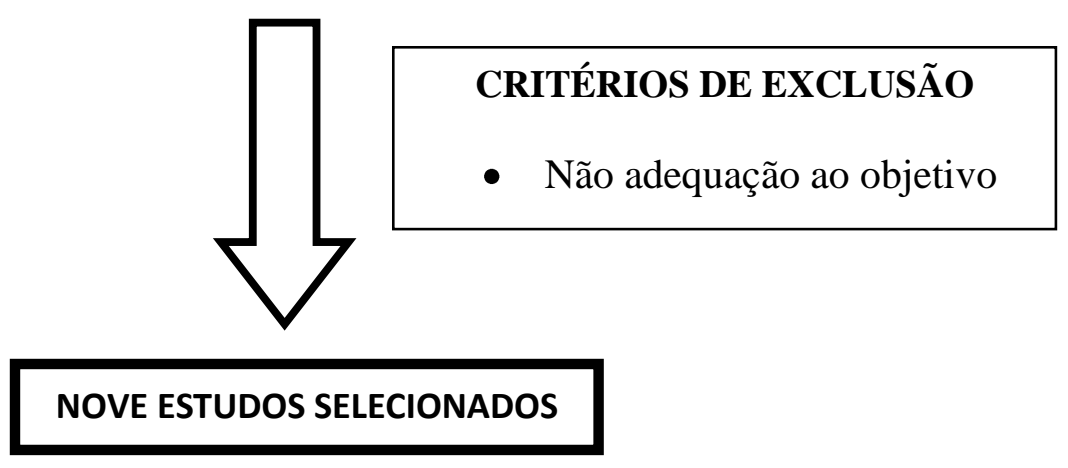

Fonte: autoria própria.

\section{RESULTADOS}

Os resultados a seguir são frutos de uma revisão de diversos trabalhos primários, entre eles: estudos in vitro, coorte, ensaio clinico randomizado, caso controle e relatos de caso.(Fig.2)

O primeiro estudo descrito na literatura que tenta demonstrar um resultado satisfatório foi um estudo in vitro realizado pelo Zhang JJ et al em 1994. Nesse trabalho em específico, o autor tenta comprovar um possível mecanismo fisiopatológico da catarata induzida por Tamoxifeno. Esse estudo demonstra que o tamoxifeno e seus derivados têm alta afinidade pelos canais de cloreto presentes no cristalino, bloqueando-os. Nesse caso, o autor explica que os canais de cloreto, presentes no cristalino têm como função manter a hidratação e transmitância normais do cristalino. Dessa forma, nesse estudo houve um bloqueio dos canais de cloreto e consequente opacidade do cristalino, gerando a catarata em concentrações clinicamente relevantes ${ }^{(5)}$.

No ano seguinte, 1995, o mesmo autor (Zhang et al), publicou um novo estudo in vitro para dar mais embasamento à sua tese apresentada no trabalho de 1994. Nesse estudo foram utilizadas culturas de lentes bovinas in vitro para comparar a possível diferença da opacidade entre o Tamoxifeno e o ICI 182,780. O ICI 182,780, um antiestrogênio esteroidal, é análogo estrutural de estrogênio de maior potência, porém de menor atividade agonista. Já o Tamoxifeno, é uma droga derivada do trifeniletileno e não relacionado ao estrogênio, possuindo uma atividade agonista parcial. Foi novamente comprovado que o Tamoxifeno causou opacidade em lentes bovinas, bloqueando os canais de cloreto destas. Em contraste, o ICI 182,780, não conseguiu suprimir a função dos canais de cloreto, somente quando sua concentração utilizada foi dez vezes maior que o estrogênio não esteroidal, o Tamoxifeno ${ }^{(6)}$.

Em relação aos estudos coorte, três estudos foram incluídos no trabalho. O primeiro foi escrito pelo Parkkari et al, em 2003, onde foi utilizada uma amostra de 60 mulheres, entre 50 e 79 anos, em pósmenopausa com câncer de mama. Essas 60 mulheres foram divididas em dois grupos com 30 
pacientes cada. Cada grupo recebeu um tipo de medicamento diferente, um grupo o Tamoxifeno e o outro o Toremifeno. Logo na primeira consulta, 16 pacientes tiveram catarata, sendo sete no grupo que utilizou Tamoxifeno e nove no grupo de Toremifeno). Além disso, três pacientes apresentaram cristais maculares, sendo uma no grupo de Tamoxifeno e duas no grupo de Toremifeno. Durante o período do estudo, 10 pacientes desenvolveram catarata (cinco em cada grupo). Drusas maculares foram diagnosticadas em cinco pacientes no primeiro exame oftalmológico (dois no tamoxifeno e três no grupo toremifeno). Não foram observados efeitos colaterais oculares graves entre os 60 pacientes com câncer de mama tratados com Tamoxifeno ou Toremifeno em um período de três anos e meio ${ }^{(7)}$. Outro estudo do tipo coorte publicado, foi do autor Costatino, em 2006, onde foi utilizada uma amostra de 10000 mulheres para analisar o risco/beneficio da terapia SERM. Nesse estudo foi demonstrado que o uso de tamoxifeno pode aumentar a ocorrência de catarata em cerca de $14 \%{ }^{(8)}$. Por fim(colocar virgula) temos o estudo do tipo coorte do autor Nichols et al, de 2014, no qual foi utilizado uma amostra de 50884 mulheres entre 35 e 74 anos de idade. Como resultado desse estudo, 49\% das usuárias de Tamoxifeno forma mais prováveis em relatar o desenvolvimento de catarata (55 usuárias de Tamoxifeno, 179 não usuárias), em comparação ao grupo de não usuárias. Além disso, o diagnóstico de catarata não foi estatisticamente significativo quando relacionado à descontinuação antes de cinco $\operatorname{anos}^{(9)}$.

Obtivemos o resultado de um estudo do tipo ensaio clínico randomizado, do autor Vogel et al, de 2009, cuja amostra foi de 19747 mulheres com 35 anos ou mais e com histórico de carcinoma lobular in situ tratado somente com excisão local e sem outros fatores de risco. Como resultado, 707 mulheres desenvolveram catarata durante o estudo, sendo divididas em dois grupos: as que tomaram Tamoxifeno (394 mulheres) e as que tomaram Raloxifeno (313 mulheres), apresentando assim um $\mathrm{RR}=0$,79. Além disso, dessas 707 mulheres, precisaram de cirurgia 260 mulheres do grupo de Tamoxifeno e 215 do grupo de Raloxifeno, sendo assim, um $R R=0,82$. Foi concluído nesse estudo que o grupo de Raloxifeno foi favorecido ${ }^{(10)}$.

Nos estudos de caso controle, foram analisados dois trabalhos. O primeiro foi do autor Paganini-Hill e Clark, de 2000, cuja amostra foi de 1297 mulheres com idade entre 57 e 75 anos. Nesse caso controle, as usuárias de termo e de longo prazo do Tamoxifeno relataram com maior frequência o desenvolvimento de catarata do que não usuárias (18,2\%, 21,4\% vs. 14,8\%, respectivamente). Dessa forma o estudo sugeriu que o uso por cinco ou mais anos de tamoxifeno aumentam o risco de catarata $^{(11)}$.O segundo caso controle analisado foi do Bradbury et al, de 2004, com uma amostra de 1107 mulheres idosas. O estudo indicou que as usuárias atuais de Tamoxifeno não apresentavam risco aumentado de catarata ( $\mathrm{OR}=1,0, \mathrm{IC} 95 \%: 0,7,1,4)$. Não houve evidência de aumento do risco com o aumento da dose cumulativa. Dessa forma os resultados deste estudo de mulheres na pós- 
menopausa fornecem pouca evidência para apoiar a hipótese de que o uso de tamoxifeno por cinco anos ou menos está associado a um risco aumentado de catarata ${ }^{(12)}$.

Um estudo do tipo relato de caso foi analisado, cujo autor foi Demirci et al, de 2003. Nesse relato foi revelado que o Tamoxifeno pode induzir um paciente a ter catarata após um pequeno tempo de uso. O autor ainda destacou que a cirurgia de catarata foi o melhor método para impedir a descontinuação do uso do Tamoxifeno (o autor explica que os benefícios do uso da droga supera seus malefícios) ${ }^{(13)}$. As evidências acerca da relação entre o Tamoxifeno e o desenvolvimento de catarata são controversas. Ainda que não unânime, grande parte dos estudos analisados revela uma efetiva opacificação do cristalino predisposta pela terapia antiestrogênica em questão (Fig.3). Alguns autores já tentaram explicar as bases fisiopatológicas do processo, mas não obtiveram resultados categóricos.

Fig.2 - Tipos de estudo.

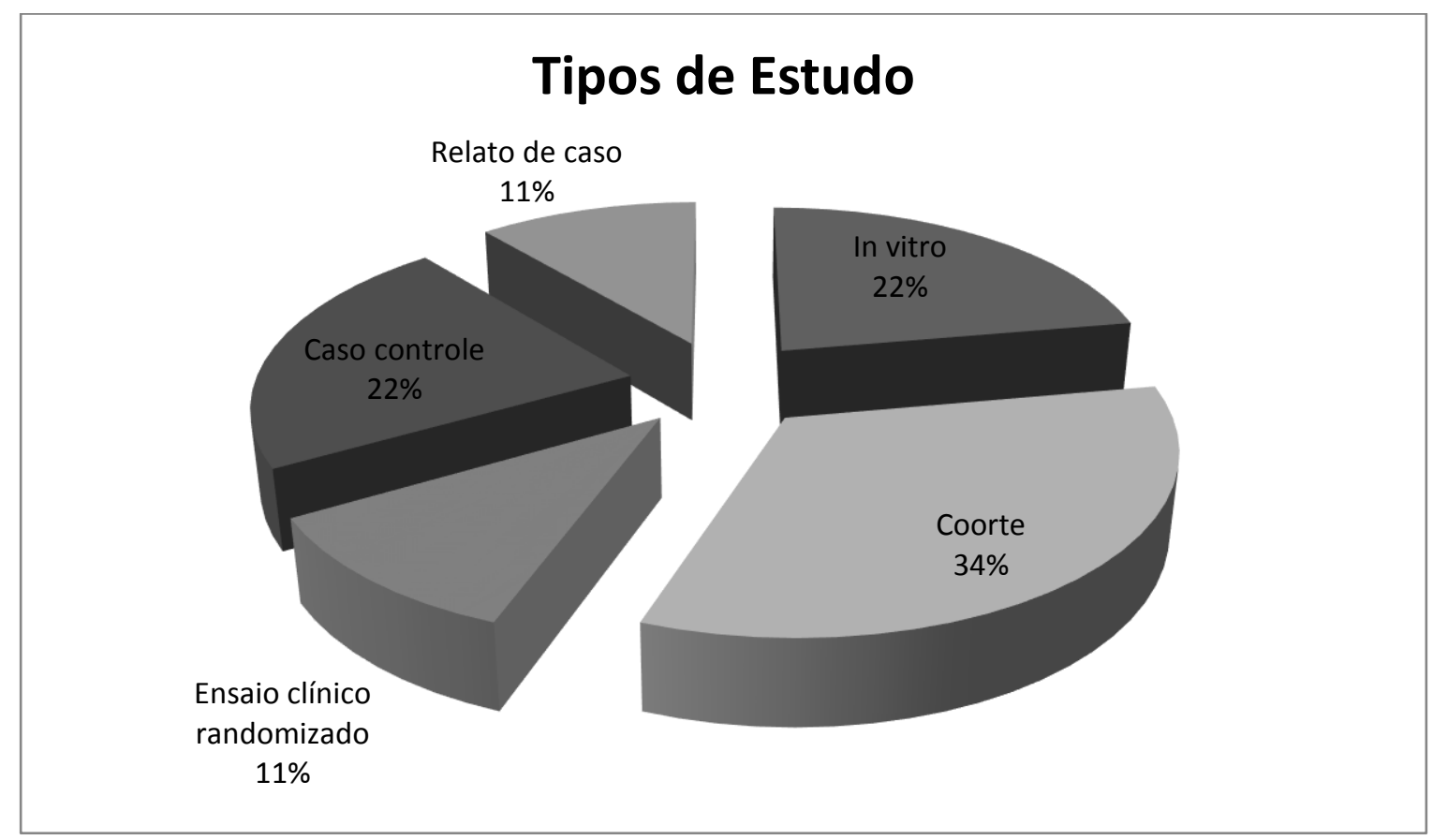

Fonte: autoria própria. 


\section{Resultado dos estudos}

- Favoráveis Não Favoráveis

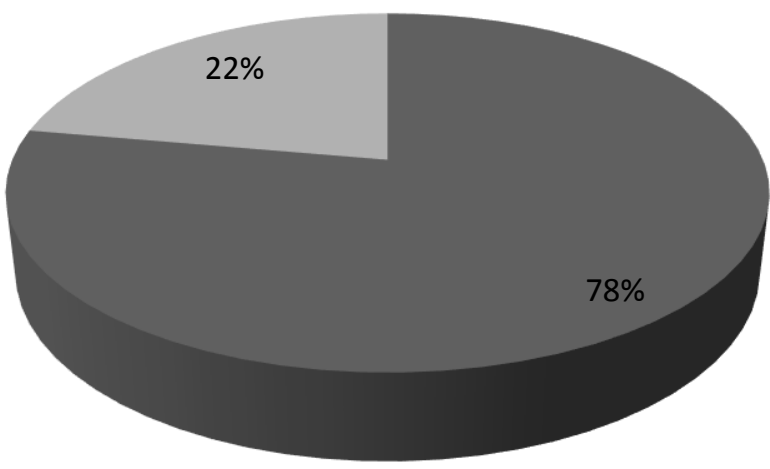

Fonte: autoria própria.

\section{CONCLUSÕES}

De fato, algumas reações oftalmológicas adversas já são bem documentadas na literatura, como é o caso da ceratopatia e retinopatia ao uso do Tamoxifeno. Contudo, a predisposição específica à catarata ainda é um tema divergente.

A literatura atual sugere que a administração do Tamoxifeno possa estar relacionada com o maior risco de opacificação do cristalino, contudo, poucos trabalhos foram realizados e muito ainda não se sabe sobre esta associação. Assim, mais estudos são necessários para a documentação de um resultado seguramente definitivo.

\section{REFERÊNCIAS}

1. Karn A, Jha AK, Shrestha S, Acharya B, Poudel S, Bhandari RB. Tamoxifen for Breast Cancer.J Nepal MedAssoc [Internet]. 31 de março de 2010 [citado 12 de outubro de 2018];49(177). Disponível em: https://www.jnma.com.np/jnma/index.php/jnma/article/view/140

2. American Cancer Society. Cancer Facts \& Figures 2008. Atlanta: American Cancer Society; 2008.

3. Kiran S. Tamoxifen: Pharmacokinetics and Pharmacodynamics. Open Access J Pharm Res [Internet]. 2017 [citado 12 de outubro de 2018];1(8). Disponível em: https://medwinpublishers.com/OAJPR/OAJPR16000143.pdf

4. GOLDSTEIN, Steven R. et al. Raloxifene use in clinical practice. Menopause, [s.1.], v. 16, n. 2, p.413-421, mar. 2009. Ovid Technologies (Wolters Kluwer Health).

http://dx.doi.org/10.1097/gme.0b013e3181883dae. 
5. ZHANG, $\mathrm{J} \mathrm{J}$ et al. Tamoxifen blocks chloride channels. A possible mechanism for cataract formation. Journal Of Clinical Investigation, [s.1.], v. 94, n. 4, p.1690-1697, 1 out. 1994. American Society for Clinical Investigation. http://dx.doi.org/10.1172/jci117514

6. ZHANG, Jin Jun et al. Lens opacification by antioestrogens: tamoxifen vs ICI 182, 780. British Journal Of Pharmacology, [s.1.], v. 115, n. 8, p.1347-1348, ago. 1995. Wiley. http://dx.doi.org/10.1111/j.1476-5381.1995.tb16622.x

7. PARKKARI, M. et al. Ocular side-effects in breast cancer patients treated with tamoxifen and toremifene: a randomized follow-up study. Acta Ophthalmologica Scandinavica, [s.1.], v. 81, n. 5, p.495-499, out. 2003. Wiley. http://dx.doi.org/10.1034/j.1600-0420.2003.00116.x

8. COSTANTINO, Joseph P. et al. Benefit/Risk Assessment of SERM Therapy. Annals Of The New York Academy Of Sciences, [s.1.], v. 949, n. 1, p.280-285, 25 jan. 2006. Wiley. http://dx.doi.org/10.1111/j.1749-6632.2001.tb04033.x

9. PARKKARI, M. et al. Ocular side-effects in breast cancer patients treated with tamoxifen and toremifene: a randomized follow-up study. Acta Ophthalmologica Scandinavica, [s.1.], v. 81, n. 5, p.495-499, out. 2003. Wiley. http://dx.doi.org/10.1034/j.1600-0420.2003.00116.x

10. VOGEL, Victor G. The NSABP Study of Tamoxifen and Raloxifene (STAR) trial. Expert Review Of Anticancer Therapy, [s.1.], v. 9, n. 1, p.51-60, jan. 2009. Informa UK Limited. http://dx.doi.org/10.1586/14737140.9.1.51

11. PAGANINI-HILL, Annlia; CLARK, Linda J. Eye problems in breast cancer patients treated with tamoxifen. Breast Cancer Research And Treatment, [s.1.], v. 60, n. 2, p.167-172, mar. 2000. Springer Nature. http://dx.doi.org/10.1023/a:1006342300291

12. BRADBURY, Brian D. et al. Tamoxifen and cataracts: a null association. Breast Cancer Research And Treatment, Netherlands, n. 87, p.189-196, 2004

13. TSAI, D-c et al. Should we discontinue tamoxifen in a patient with vision-threatening ocular toxicity related to low-dose tamoxifen therapy? Eye, [s.1.], v. 17, n. 2, p.276-278, mar. 2003. Springer Nature. http://dx.doi.org/10.1038/sj.eye.6700317. 